\title{
DESIGN AND "RESTORATION": THE ROOTS OF ARCHITECTURE PORJECT FOR THE BUILT
}

\author{
Christian Campanella \\ Politecnico di Milano - Dep. ABC - ( christian.campanella@polimi.it)
}

Commission

KEYWORDS: design, surveying, photogrammetry, restoration, conservation, project

\begin{abstract}
:
It is absolutely essential now to prepare a project of pre-critical understanding of the building which will be the object of action, free from preconceived notions of value, fully committed to the implementation of a variety of useful and indispensable to determine that operating margin and freedom that each architecture has not being linked uniquely to the precise original function. This is the margin that you can leverage to implement the architectural project for the built that mutuerà inside knowledge, conservation and innovation at the same time. The binomials in the lead paragraph as all sides of the same coin that merge into one integrated design process that takes charge of design including those different styles of drafting of survey (in all aspects) of an existing architecture.
\end{abstract}

\section{Sketch and "restauration": to the roots of the project of architecture for the built one.}

Sketch and restauration: a binomial strongly certificate that in the years it is radically evolved both in the contents (technical, theoretical and methodological) and in the operational fit strengths of intervention. Relief and maintenance of the buildings, knowledge and project, are the today's interdisciplinary joinings to which we cannot delegate in to face an any intervention of architectural planning that involves, also only partially, that immense patrimony to be safeguarded (built and not), that we define well cultural assets.

A patrimony continually and constantly not only attacked by the carelessness, from the degrade and from the abandonment, but very often from a formality of intervention on the built one distant from one by now attested methodological routine of approach to the project tied to the knowledge, to the preliminary reading, to the evaluation of the strength state of health of the manufactured article, to the full understanding of its character.

And' absolutely essential today to predispose a project of critical pre-understanding of the building that will be object of intervention to desk, deprived from preconceived attributions of value, addressed to the mass in action of varied useful and essential activities to determine that operational border and of liberty that every architecture possesses not being tied up to the precise native function. And' on this border that lever can be made for setting in action the project of architecture for the built that it will assimilate to its inside knowledge, maintenance and innovation at the same time. The binomial ones fall in head to the paragraph being all faces of the same medal that melt him in an only process of integrated planning that he makes load of varied styles of editing design including those some survey (in all itsr aspects) of an existing architecture.

The demand of the debugging of a protocol of relief of the existing one, sinks far the roots in the time. The binomial maintenance and knowledge, even though covertly it already renders explicit him through a lot of nineteenthcentury norms, you define to guardianship of the historical buildings. Among the so many the Rule for the Committee Ausiliarie of Belle Arti of the Pontifical State, emanated in 1821, that it is expressed against the bad ones and the useless restaurations, forcing to the predisposition of accurate reliefs, before proceeding to the execution of any intervention: "The respective Committee will get in all the ways to make to raise these plants and these sketches. In them it will be enough that is well marked, and drawn that it exists, without taking intrigues to make up for the lacks, while to the times hill suppositions are given the most beautiful, but truer sketches."

And' Camillo Boito, in 1865, to want you unite the disciplines of the restauration and the sketch what subjects of teaching to the second and third year of the school of Application in Milan, useful to achieve (in the three years) the diploma of civil architect. The demand is real, but wish almost exclusively resulted since that time tied up to the aspect document to be able to put her the best practices of the into effect "restauration", almost always unhooked by a fit strength of safeguard that cannot be exempted by the predisposition of a project of architecture intended as result of the interaction among maintenance, innovation and use. A perpetuated wish and defined through for long time all the Italian Papers of the Restauration beginning from that of 1883 where to the art 6 are underlined the importance of the photo before, during and 
after the jobs, of the relief and of the sketch which orchestrates essential useful to identify remaking, consolidations, changes removals, destructions. The same indications appear to the art 11 of the 1932 Paper, to the art. 9 of the Instructions for the 1938 monuments use, to the art. 16 of the Paper in Venice, to the art. 5 and to the enclosure b) of the Italian Paper of the 1972. And' the analogical and digital instrumentation that after sometime it constantly implements the bond between relief and project of maintenance, in special way through the evolution of the instrumentation of indirect (total stations, TLS, terrestrial photogrammetry) relief. In special way the contribution of the photo assumes in the years value which I document metric of the more and more "real" taking space field of the architectural reading, of the documentation, of the representation. It doesn't replace him with the sketch, or not from immediately, but it becomes in the time an important tool so much to give impulse and development to the relief photogrammetric beginning from 1851 straight more and more, through intersections in ahead in which the measures were gotten by the frames, even though with notable errors caused by the distorsion of the photographic objectives. The job and the constant search of a methodology able to numerically reproduce the dimensions of an object through the photo actually crossed the whole second halves the 800 and the first halves 1900 to the advent of the electronics that, beginning from the years ' 60 of 900, gave a lot of impulse to the photogrammetry with equipments with great ability of calculation and small dimensions, actually to the advent and the development of the digital era.

Still today the binomial one "relief and restauration" it withstands inside the so many Laboratories of Restauration of our Italian Faculties of Architecture, but the concept of such interrelation has widened by now, arriving to adopt new terminologies (what relief and maintenance, survey and project of intervention for the existing building) having the meanings and the finalities of it well clear: the survey what together of practical and analytical operations to perform him to determine the specificities of an object; the survey which picked up systematic of individual observations of a collective phenomenon to draw dates statistic of it that concern the same phenomenon. Survey therefore what operational action directly performed, as a document complex, not end to himself, able to pick up and to transmit data base of the cognitive trial of the building. Point of departure of a methodological run that it takes sprout from the listening of the factory, of the subject of which it is composed, of the problems that bother it, so that to draw indications of it useful to the predisposition of the project of intervention, to transmit in use what today it results abandoned or under used.

\section{Survey and project.}

Unlike what can happen in a new building, in the preparation of the project of intervention for the existing building, the manufactured article assumes the role of actor protagonist, carrier of a lived of whom witness is directed. As such, functionalized can be considered and valorized only through a "listening" careful.
To listen to the subject, a place, a simple or complex architecture seems an insane action, almost incomprehensible imagining the world of the existing building as immovable property atonic place of the way of living. So it is not, the subject is in continuous movement, it is in constant vibration, uninterruptedly transformed by lights and shades that minute is run after after minute, from the use in continuous mutation, to flow and to flow out some man that marks it, consumes it, transforms it instant after instant. All it takes is observing for lowering in the reality of a place, it needs enter to make part gathering of it of it the humor that is it really, developing in first analysis an activity of listening that it hardly succeeds selective in how much incapable to accomplishedly define of it and physically the contours. The reading that achieves won't be able not to keep in mind of it of the strong empathy (positive or negative) that this phase must be able to activate with the place object of job. The listening can perhaps be defined the antechamber of entry to the reading of the physical reality, borrowed by the structural and interpretative filter of the perceptive reality that we obviously succeed in gathering and to understand through the sensitive organs.

It enters so in game a moment interlocutory carrier for its nature of two consequent phases of analysis: an initial "passive" and the other, necessarily following, "active." The first moment acquires a value maieutica and therefore as such, not to lose any information, doesn't exclusively assemble him on the single manufactured article, but it is necessarily expressed, at least in a first phase, to ampler staircase. The relief, before defining the characteristics metric, morphological and materics of a building must be dealt with the context up to the urban staircase, in which the object is inserted, of which it is active part. This ample approach contributes besides (often in conclusive way), to the definitive choice of a possible use, conferring to the option territorial legitimacy. The object will speak so of itself, of its around and of the relationships away, street more and more external through which can have arrived to acquire value of system in urban-territorial relationships, as instead you can underline how much these relationships have been denied. Never as in this case necessity is underlined to proceed through a deductive reflection that allows to reach, through a mediate inference, from a general state to a particular conclusion. The first moment, if assumed with the maximum respect for the manufactured article, also making itself load of the intrinsic characters to the planner, it can bring to answers of intervention from the intense contents, formal, technological, compositive, distributive. It goes therefore considered as a real occasion of analysis, promoter often significant design ideas. An analysis that dilates it, to the continuous search of information (but also of stimuli and suggestions), through the historical search, the diagnostic preventive, the scientific investigation, the identification and quantification physical-chemistry. All operations preparatiry to the planning, that it ask for the involvement of specific professionalism able to elaborate data, analysis, schedature, reliefs type qualitative and quantitative. An 
unbelievable number of indications to be calibrated and to select, that become useful only once interrelate the one to the other bottom the control of a careful and aware direction.

The results of the country of diagnostic survey allow us to take full conscience of the strength state of any building, furnishing us indications on its geometric characteristics, physics and materics, on his proper real static abilities, performance, morphological and distributive.

They identify every time unusual quality, always defined by different factors, in degree to pilot the design choices, confirming the oneness of every single building. The continuous listening, the reading of the subject and the signs that brings engraved, the understanding and the taking of action of the formal complexity, architectural and tipologicas of a manufactured article certainly amplify a degree of confidence with the useful circumferentor / planner to fully understand its character.

You underlines as the necessity to have to intervene on a degraded building makes this careful phase decisive "passive" of relief. Decisive in how much, a final synthetic image must define, choices must be made and to take decisions on thing and as to purely return not only the useful in relief data from the quantitative point of view, but also what clear qualitative expression, performance, morphological of an architecture that we obstinately want to preserve and to the meantime to innovate returning it to the contemporary use.

Well it renders explicit it in this way as the final objective of the relief both that to meet in an operational project, where only the hold relationship among the two phases comes to qualify her both, conferring them a specific and independent disciplinary character, always corrections subject and adjustments that become meaningful really for their systemic value. In this context the characteristic of their continuous becoming finds space of expression and meaning of intents.

To the first moment it will evidently follow the phase "active" in which must be made to yield the precedent listening, to allow this point a graphic restitution the most complete possible, that to the intervention.

The staircase of restitution to use him, in the field of the maintenance of the built one, it doesn't represent a secondary choice, already constituting a variable to the precision and the close examination possible. You so immediately reaches the first compromise to which you then to wheel all the others, what: the type of graphics to be gotten a job, (both for the geometric relief and for that pathological and materic relief) the staircase, the choice of the particular ones to be underlined, the definition of the photographic restitution, the quantity of the acquirable information. And still, the use of new tools of survey expeditious, software specific and of the electronic elaboration in continuous evolution, but not yet completely in degree (in the field of the "architectural restauration") to manage the vast complexity of the data noticed to correlate him among them. Today, the geomatica is for instance, able to furnish a great massive structure of information allowing us to reproduce sophisticated three-dimensional models of whole buildings or part of them more and more. And' a tool still in full evolution that can certainly reveal useful in the and for the development of the project for the existing building. A project that obviously you cannot be exhausted in the simple treatment of the surfaces, but that you must go over dealing itself with the tied up matters to the improvement and the consolidation of the structures, of the use, of the new function, of the relationship with the new one that inevitably it will go to contaminate the building object of intervention. A relationship that today we plan and we dimensionally represent or three-dimensionally unhooked by the facies of the factory for the most part producing abstract models if not nearer rendering to a cartoon that to the reality.

The tied up evolution then to the systems of survey and restitution (photogrammetry, laser scanners, photographic straightening, 3D) it is developing through also the employment of the BIM (Building Information Modelling), been born and developed its for the planning ex novo, but to appraise, to experiment and to also apply to the existing housebuilding being the representation of a model of different data of a building reported to the different disciplines that define it. The contained data in the model, in the case of degrade existing architectures, in comparison to a new construction, they multiply him in how much they define all the information concerning a specific component of the building in matter rich of variable specific case by chance. And' an evolution, not certainly simple, to wish being besides able the system to manage The cycle of life of the work from the desgin phase, through the phase of realization, up to that of use and maintenance.

The type of the factory, its character, its subject, its morphology they ask even more however, they ask solutions that only the same factory is able to produce founded upon its volumes, its spaces its distribution that we are able to gather and to absorb only and exclusively from its investigated roots and you represent through the survey / revelation of the whole and only palinsest that defines it in the space.

From these bases, this second phase "active" it will assume away, by strong design value resulting what first fit strength of safeguard, able to dictate the rules, before, during and after possible operations of maintenance, qualification and use to perform him on the existing architetcture.

The minute reading, the knowledge of the folds of every single building they will become the true points of departure and strength of the project of architecture for the existing building, it is the development of a sort of architectural SWOT able to gather points of strength and weaknesses, opportunity and threats that go to define its vocation, the real functional potentiality, the border besides which it is easy to slip, from a side conferring to the object an extreme dowry of character that can conduct it to a sort of hibernation, actually to the revival than it is lost, to the reconstruction in style of the "good season" (the happiest) of its existence. From the other to assume him as pretext to underline and to implement unlikely contemporary (next to, in overlap, in substitution) realizations, 
true protagonists of the project of intervention, strongly self-referenzial and you rarely think of continuity with the built one assumed (from immediately) by the planners as simple private soldier, useful to reach a well precise purpose already fixed well before the departure, mean and not end for the project of the new one.

The manufactured article and the planner through the relief have therefore an extreme need to listen to him, to compare him to learn to know him, up to the point in which the phase final design will arrive to assume a connotation usual great naturalness. This last datum, when it is centered as objective, it contributes to underline the pregnancy that the relief as a whole of its two phases, it has for the whole project of architecture for the built one. The "naturalness" of the design idea that it derives it won't be never a banal solution of it and discounted really, because the listening will be tall and qualified anymore, the inferred project will qualify anymore him as "the only one" possible, the most correct and calibrated for that datum building.

Only investigating to fund the particularities of every single building, able you can be been to operate without performing heavy and irreversible tamperings, putting to regime a project of architecture for the built that from the same built, it takes form and new value in use. 